\title{
Impro ved Optimal Wighted Links Algorithms
}

\author{
Ovidiu Daescu \\ Department of Computer Science, \\ University of Texas at Dallas, \\ Richardson, TX 75083, USA \\ daescu@utdallas.edu
}

\begin{abstract}
In this paper, we present improved algorithms for computing an optimal link among weighted regions in the 2-dimensional (2-D) space. The weighted regions optimal link problem arises in several areas, such as geographic information systems (GIS), radiation therapy, geological exploration, environmental engineering and military applications. Our results are based on a (more general) theorem that characterizes a class of functions for which optimal solutions arise on the boundary of the feasible domain. A direct consequence of this theorem is that an optimal link goes through a vertex of the weighted subdivision. We also consider extensions and present results for the 3 -D case. Our results imply significantly faster algorithms for solving the $3-\mathrm{D}$ problem.
\end{abstract}

\section{Introduction}

We consider the 2-dimensional (2-D) weighted regions optimal link problem: Given a planar subdivision $R$, with $m$ weighted regions $R_{i}, i=1,2, \ldots, m$, and a total of $n$ vertices, find a link $L$ such that: (1) $L$ intersects two specified regions $R_{s}, R_{t} \in R$ and (2) the weighted sum $S(L)=\sum_{L \cap R_{i} \neq \phi} w_{i} * d_{i}(L)$ is minimized, where $w_{i}$ is either the (positive) weight of $R_{i}$ or zero and $d_{i}(L)$ is the length of $L$ within region $R_{i}$. Depending on the application, the link $L$ may be (a) unbounded (i.e., a line): the link $L$ "passes through" the regions $R_{s}$ and $R_{t}$; (b) bounded at one end (i.e., a ray): $R_{s}$ is the source region of $L$ and $L$ passes through $R_{t}$ and (c) bounded at both ends (i.e., a line segment): $R_{s}$ is the source region of $L$ and $R_{t}$ is its destination region. Let $R_{L}$ be the set of regions $\left\{R_{i_{1}}, \ldots, R_{i_{k}}\right\}$ intersected by a link $L$. Then, $w_{i_{1}}$ and $w_{i_{k}}$ are set to zero. This last condition ensures that the optimal solution is bounded when a source (and/or a destination) region is not specified (cases (a) and (b)) and allows the link to originate and end arbitrarily within the source and target regions (cases (b) and (c)).

The weighted regions optimal link problem arises in sev eral areas sudh as GIS, radiation therapy, stereotactic brain surgery, geological exploration, environmental engineering and military applications. For example, in military applications the weight $w_{i}$ may represent the probability to be seen by the enemy when moving through $R_{i}$, from a secured source region $R_{s}$ to another secured target region $R_{t}$. In radiation therapy, it has been pointed out that finding the optimal 
choice for the link (cases (a) and (b)) is one of the most difficult problems of medical treatment optimization [3]. In what follows, we will discuss case (c) of the problem that is, we want to compute a minimum weighted distance between $R_{s}$ and $R_{t}$. The other two cases can be easily handled in a similar way.

Previous work. There are a few results in computational geometry that consider weighted region problems, for computing or approximating optimal shortest paths between pairs of points. We refer the reader to $[1,17,18,20]$ for results and further references. The optimal link problem however, has a different structure than the shortest path problem and its complexity is not known yet. In general, a $k$-link optimal path may have a much larger value than a minimum weighted length path joining the source and the target regions. The bicriteria path problem in a graph (does there exists a path from $s$ to $t$ with length less than $L$ and weight less than $W$ ) is known to be NP-hard [14]. For the related problem of computing an Euclidean shortest path between two points within a polygonal domain ( 1 and $\infty$ weights only), constrained to have at most $k$-links, no exact solution is currently known. A key difficulty is that, in general, a minimum link path will not lie on any simple discrete graph. We refer the reader to [19] for more details and references on optimal path problems.

Note that case (c) of the link problem asks to find the minimum weighted distance $d_{s t}$ between $R_{s}$ and $R_{t}$. From the definition of the link problem, the weights $w_{s}$ and $w_{t}$ of $R_{s}$ and $R_{t}$ are zero, and thus $d_{s t}$ is also the optimal weighted bridge between $R_{s}$ and $R_{t}$. We mention that for the unweighted case, all the weights are one and optimal linear time algorithms are known for finding the minimum distance or bridge $[4,16]$.

Important steps toward solving cases (a) and (b) of the link problem have been first made in [7], where it has been proved that the 2-D problem can be reduced to a number of (at most $O\left(n^{2}\right)$ ) global optimization problems (GOPs), each of which asks to minimize a 2-variable function $f(x, y)$ over a convex domain $D$, where $f(x, y)$ is given as a sum of $O(n)$ fractional terms. Very recently, efficient parallel solutions for the optimal link problem have been reported in [10], where it has been shown that, if at most $n$ processors are available, all GOPs can be generated using $O\left(n^{2} \log n\right)$ work. Since the GOPs can be efficiently generated $[7,10]$, most of the time for computing an optimal link will be spent solving the GOPs. Using 2-D global optimization software to solve the GOPs may be too costly, however. In [8], it has been pointed out that, for simpler functions such as sum of linear fractionals (SOLF), while commercially available software performs well for the $1-\mathrm{D}$ case, it has difficulties for the 2 -D case. The experiments in [7] have shown that the optimal solution goes through one or even two vertices of the subdivision $R$ (i.e., it is on the boundary or at a vertex of the feasible domain) or it is close to such vertex or vertices (which may be due to numerical errors). Following those experiments, it remained an open problem to prove or disprove that a global optimal solution can always be found on the boundary of the feasible domain.

Our results. In this paper, we affirmatively answer the question above. More specifically: (1) We prove that, given a global optimization problem instance as 


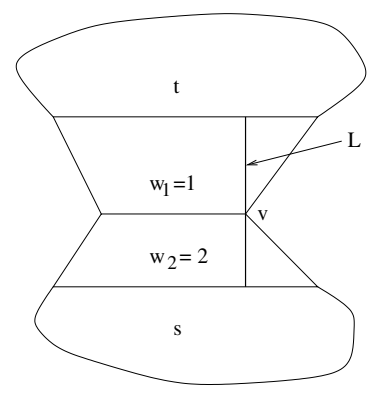

Fig. 1. An optimal link $L$ goes through at most one vertex.

above, the 2 -variable objective function attains its global optimum on the boundary of the feasible domain, thus reducing it to a 1-variable function. Accordingly, an optimal link goes through a vertex of the subdivision $R$. For this, we give a theorem that characterizes a class of functions for which optimal solutions can be found on the boundary of the feasible domain. Since some special instances of the 2-D SOLF problem fall in this class [8], significant speed-up over the general SOLF algorithms are possible for these instances. On the other hand, it is not hard to construct examples for which the optimal solution does not go through two vertices of the subdivision (i.e., it is in the interior of some boundary edge of the feasible domain). A simple example is given in Figure 1. (2) Our solution for case (c) of the link problem results in an efficient algorithm for finding the minimum weighted distance (and the minimum weighted bridge) $d_{s t}$ between $R_{s}$ and $R_{t}$. (3) Using the same models of computation as in [10], very simple parallel algorithms for generating the GOPs can be developed. While matching the time/work bounds in [10], these new algorithms are expected to be faster in practice, since they do not require arrangement computation. Due to space limitation, we leave these results to the full paper. (4) We show that our results can be extended to the 3 -D case. Consequently, 4-variable objective functions over 4-D domains are replaced with 2-variable functions over 2-D domains, and a relatively simple data structure can be used to generate the corresponding 2-dimensional optimization problems.

Some consequences of our results are: (1) Tremendous speed-up in solving the 2-D optimal link problem, when compared to the algorithms in [7] (and possible those in [8]): solve $O\left(n^{2}\right) 1-\mathrm{D}$ global optimization problems as opposed to $O\left(n^{2}\right) 2-\mathrm{D}$ global optimization problems in [7]. (2) Commercially available software such as Maple may be used even for large values of $n$ to derive (local) optimal solutions. (3) Our results for the 3 -D case (a) make an important step toward efficient solutions for the 3 -D version of the optimal link problem and (b) can be used to obtain efficient solutions for the 3 -D version in a semi-discrete setting. (4) Efficient approximation schemes can be developed, by discretizing the solution space. The inherent parallelism of such algorithms may be exploited to obtain fast, practical solutions. 


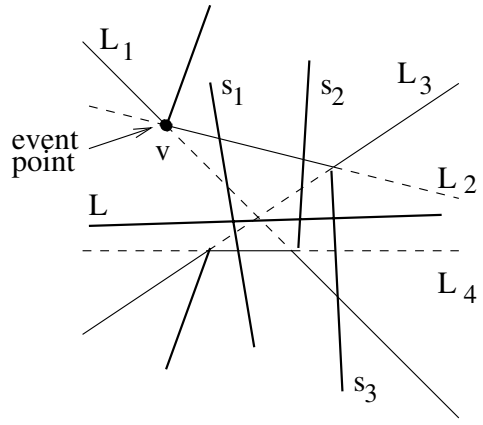

(a)

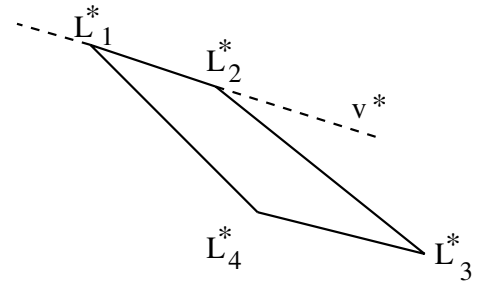

(b)

Fig. 2. (a) A link $L$ intersecting $s_{1}, s_{2}$ and $s_{3}$ and (b) The corresponding dual cell.

\section{Background and notations}

In this section we introduce some notations and useful structures. Consider a planar weighted subdivision $R$, with a total of $n$ vertices. Let $L$ be a link intersecting the source and target regions, $R_{s}$ and $R_{t}$. Let $S$ be the set of line segments in the subdivision $R$ and let $S_{s t}=\left\{s_{i_{1}}, s_{i_{2}}, \ldots, s_{i_{k}}\right\}$ be the subset of line segments in $S$ that are intersected by $L$. Consider rotating and translating $L$. We say that an event $e_{v}$ occurs if $L$ passes a vertex $v$ of $R$. Such an event corresponds to some line segments (with an endpoint at $v$ ) entering or leaving $S_{s t}$ (see Fig. $2(\mathrm{a})$ ). As long as no event occurs, the formula describing the objective function $S(L)$ does not change and has the expression $S(L)=\sum_{i=i_{1}}^{i_{k}-1} w_{i} * d_{i}$. Here, $d_{i}$ is the length of $L$ inside region $R_{i}$. That is, $d_{i}$ is the length of a line segment with endpoints on segments $s_{i}$ and $s_{i+1}$, where $s_{i}$ and $s_{i+1}$ are on the boundary of $R_{i}$. We refer the reader to $[7,9]$ for more details.

Let $H=\left\{l_{1}, l_{2}, \ldots, l_{n}\right\}$ be a set of $n$ straight lines in the plane. The lines in $H$ partition the plane into a subdivision, called the arrangement $A(H)$ of $H$, that consists of a set of convex regions (cells), each bounded by some line segments on the lines in $H$. In general, $A(H)$ has $O\left(n^{2}\right)$ faces, edges and vertices and it can be optimally computed in $O\left(n^{2}\right)$ time and $O(n)$ space, by sweeping the plane with a pseudoline [13]. If one is interested only in the part of $A(H)$ inside a convex region, similar results are possible [2].

Assume the link $L$ is a line, given by the equation $y=m x+p$ (case (a) of the optimal link problem). Then, by using the well known point-line duality transform that preserves the above/bellow relations (i.e., a point $q$ above a line $l$ dualizes to a line that is above the dual point of $l$ ), all lines intersecting the same subset of segments $S_{s t} \in S$ correspond to a cell in the dual arrangement $A(R)$ of $R$ (see Fig. 2 (b)). Here, $A(R)$ is defined by the line set $H_{R}=\left\{l_{1}, l_{2}, \ldots, l_{n}\right\}$, where $l_{i} \in H_{R}$ is the dual of vertex $v_{i} \in R$. The case of a semiline (case (b) of the link problem), and that of a line segment can be reduced to that of a line, by 
appropriately maintaining the set of line segments intersected by $L$ and dropping those that arise before a segment in $R_{s}$ or after a segment in $R_{t}$. This can be done sequentially in amortized constant time, by using the data structures in [7, 9]. Thus, one can produce the $O\left(n^{2}\right)$ global optimization problems (GOPs) by sweeping the arrangement $A(R)$ : for each GOP, the feasible domain corresponds to some cell in $A(R)$, and the objective function is a 2-variable function defined as a sum of $O(n)$ fractional terms.

Simplifications. Rather than generating and sweeping the entire arrangement, observe that it is enough to compute the portion of $A(R)$ inside a (possibly unbounded) region $D_{s t}$, bounded by two monotone chains: $D_{s t}$ is the dual of the set of lines intersecting both $R_{s}$ and $R_{t}$. Thus, computing the cells of the arrangement defined by $A(R)$ that correspond to set of lines intersecting both $R_{s}$ and $R_{t}$ reduces to computing an arrangement of line segments in $D_{s t}$. This can be done in $O(n \log n+k)$ time [6], resulting in an output sensitive, $O(n \log n+k)$ time algorithm for generating the GOPs, where $k$ is the total complexity of the feasible domains of the GOPs and can be $O\left(n^{2}\right)$ in the worst case. For case (c) of the link problem, observe that all possible links between the source region $R_{s}$ and the target region $R_{t}$ can be found in the subdivision $R^{\prime}=R \cap C H\left(R_{s}, R_{t}\right)$, where $C H\left(R_{s}, R_{t}\right)$ is the convex hull of $R_{s} \cup R_{t} . C H\left(R_{s}, R_{t}\right)$ and $R^{\prime}$ can be easily computed in $O(n \log n)$ time and we assume this "clean-up" computation done as a preprocessing step.

Lemma 1. Given a planar weighted subdivision $R$ with convex regions and a total of $n$ vertices, all the GOPs associated with the optimal link problem can be generated in $O(n \log n+k)$ time, where $k$ is the complexity of the feasible domains of the reported GOPs.

In the lemma above, the convexity is required in order to apply the data structures in $[7,9]$ and obtain constant amortized time for generating a GOP. If the regions of $R$ are not convex, $R$ can be easily triangulated to satisfy the convexity condition.

\section{GOP Analysis}

As stated earlier, the optimal link problem can be reduced to solving a number of (at most $O\left(n^{2}\right)$ ) global optimization problems, where each GOP is generally defined as $\min _{D} \sum_{i=1}^{k-1} w_{i} * d_{i}$ or equivalently [7]

$$
\min _{(x, y) \in D} f(x, y)=\sqrt{1+x^{2}} \sum_{i=1}^{k} \frac{a_{i} y+b_{i}}{x+c_{i}}
$$

where $D$ is a (convex) 2-D domain, $a_{i}, b_{i}$ and $c_{i}$ are constants and the variables $x$ and $y$ are the defining parameters for the link $L$ (i.e., slope and intercept). Without loss of generality, we assume the denominators are positive over $D$. Observe that, with the $L_{1}$ and $L_{\infty}$ metrics, the square root factor vanishes, 
resulting in a special case of the 2 -D sum of linear fractionals (SOLF) problem [8]. Experimental results in [7] have shown the following fenomenon: the optimal solution for (1) is found on, or close to (but this may be due to numerical errors) the boundary of the feasible domain. It has remained an open problem to prove or disprove that a global optimal solution can always be found on the boundary of the feasible domain (i.e., it goes through a vertex of the subdivision $R$ ). In this section we consider a GOP as defined above and prove that indeed the 2 -variable objective function attains its global optimum on the boundary of the feasible domain, thus reducing it to a 1-variable function. Accordingly, the optimal link goes through a vertex of the subdivision $R$.

Let $D$ be a bounded, 2-dimensional domain and let $g(x, y)$ be a 2-variable function over $D$ defined by the equation

$$
g(x, y)=h(x) \sum_{i=1}^{k} \frac{q_{i}(y)}{r_{i}(x)}
$$

and such that $Q(y)=\sum_{i=1}^{k} a_{i} q_{i}(y)$ is a monotone function over D, for any positive constants $a_{i}, i=1, \ldots, k$. Without loss of generality, we assume that $r_{i}(x)$ is positive over $D$, for all $i=1,2, \ldots, k$. We then have the following theorem.

Theorem 1. Given a function $g(x, y)$ defined as above, $\min _{(x, y) \in D} g(x, y)$ can be found on the boundary of the feasible domain $D$.

Some of the cells associated with the link problem that appear on the boundary of $D_{s t}$ may not be convex. It is important to observe that Theorem 1 can be applied to such domains since it does not require the feasible domain to be convex (or even piecewise linear).

The lemma bellow settles the open problem from [7].

Lemma 2. The optimal solution for a GOP instance associated with the 2-D optimal link problem can be found on the boundary of the feasible domain.

Proof. It is enough to show that a GOP instance satisfies the framework of Theorem 1, from which the proof follows. Consider a GOP associated with the optimal link problem. We can set $h(x)=\sqrt{1+x^{2}}$ and $q_{i}(y)=a_{i} y+b_{i}$. For a given value of $x$,

$$
Q(y)=\sum_{i=1}^{k} q_{i}(y) \frac{1}{r_{i}(x)}=\sum_{i=1}^{k}\left(a_{i} y+b_{i}\right) \frac{1}{r_{i}(x)}=y \sum_{i=1}^{k} \frac{a_{i}}{r_{i}(x)}+\sum_{i=1}^{k} \frac{b_{i}}{r_{i}(x)}
$$

is a linear function and thus monotone. Then, the framework of Theorem 1 holds.

Corollary 1. The 2-D optimal link problem has an optimal solution (i.e., link) that goes through a vertex of the subdivision $R$. 
Let $e_{j}$ be a boundary edge of $D$ and let $v_{j}\left(x_{j}, y_{j}\right)$ be the vertex of $R$ that has the line supporting $e_{j}$ as its dual. Edge $e_{j}$ corresponds to links going through vertex $v_{j}$ and, for $e_{j}$, the initial 2-dimensional GOP reduces to the following 1-dimensional GOP:

$$
\min _{x \in D_{x}} f_{x}(x)=\sqrt{1+x^{2}}\left(d_{0}+\sum_{i=1}^{k} \frac{d_{i}}{x+c_{i}}\right)
$$

where $d_{0}, c_{i}$ and $d_{i}, i=1,2, \ldots, k$, are constants, $D_{x}$ is the interval on the $\mathrm{X}$-axis bounded by the x-coordinates of the endpoints of $e_{j}$ and $k$ is $O(n)$ in the worst case. Summing over all boundary edges of $D$, a 2-D GOP is reduced to $O(r) 1-\mathrm{D}$ GOPs, where $r$ is the boundary complexity of $D$. Since we solve a 1-D GOP for each edge of $A(H)$, we solve $O\left(n^{2}\right) 1$-D GOPs overall, rather than $O\left(n^{2}\right) 2-\mathrm{D}$ GOPs in the previous solutions $[7,9]$.

There are a few things to note here. First, besides reducing the dimension of a GOP, the new objective function is as simple as the initial one (in fact, $O(k)$ fewer arithmetic operations are necessary to evaluate the new objective function). Having a simple objective function is important: for $n=1000$, assuming an average of 100 iterations per GOP, $O\left(n^{2}\right)$ GOPs each with $O(n)$ terms in the objective function would easily amount for a few teraflops, even without counting the additional computation required by the global optimization software (e.g., computing the derivatives, etc.). Second, commercially available software such as Maple may be used even for large values of $n$ to derive (local) optimal solutions. Further, when using similar optimization software as in $[7,8]$, we expect order of magnitude speed-up and increased accuracy in finding the optimal solutions. Third, observe that our results are more general and could be applied to other problems. For instance, we do not require convexity of the feasible domain $D$ and the boundary of $D$ may be described by some bounded degree functions given in explicit form, rather than a piecewise linear function.

If the $L_{1}$ or $L_{\infty}$ metric is used instead of the $L_{2}$ metric, the objective function of a GOP has been shown in [8] to reduce to $f(x, y)=C+\sum_{i=1}^{k} \frac{a_{i} y+b_{i}}{x+c_{i}}$, where $C, a_{i}, b_{i}$ and $c_{i}, i=1,2, \ldots, k$, are constants. In [8], a fast global optimization algorithm has been proposed for solving the 1 and 2-dimensional sum of linear fractionals (SOLF) problem:

$$
\max _{\left(x_{1}, \ldots, x_{d}\right) \in D} f\left(x_{1}, \ldots, x_{d}\right)=\sum_{i=1}^{k} \frac{n_{i}\left(x_{1}, \ldots, x_{d}\right)}{d_{i}\left(x_{1}, \ldots, x_{d}\right)}
$$

where $n_{i}\left(x_{1}, \ldots, x_{d}\right)$ and $d_{i}\left(x_{1}, \ldots, x_{d}\right)$ are linear $d$-dimensional functions. Their experimental results show that the proposed SOLF algorithm is orders of magnitude faster on 1-dimensional SOLFs than on 2-dimensional ones.

Lemma 3. A 2-dimensional SOLF problem of the form $\min _{(x, y) \in D} f(x, y)=$ $\sum_{i=1}^{k} \frac{a_{i} y+b_{i}}{d_{i} x+c_{i}}$ has an optimal solution on the boundary of the feasible domain. 
Consider a boundary edge $e_{j}$ of the feasible domain $D$ and let $y=a x+b$ be the equation of the line supporting that edge. Then, the initial 2-dimensional SOLF reduces to the following 1-dimensional SOLF:

$$
\min _{x \in D_{x}} f_{x}(x)=d_{0}+\sum_{i=1}^{k} \frac{d_{i}}{x+c_{i}}
$$

where $d_{0}, c_{i}$ and $d_{i}, i=1,2, \ldots, k$, are constants and $D_{x}$ is the interval on the $\mathrm{X}$-axis bounded by the $\mathrm{x}$-coordinates of the endpoints of $e_{j}$. Note that convexity of $D$ is not required and the boundary of $D$ may be described by some bounded degree functions.

Then, for 2-dimensional SOLF problems as in Lemma 3, one can use the 1-dimensional version of the SOLF algorithm, and thus tremendous speed-up over the general 2-dimensional SOLF algorithm is possible for those instances. We refer the reader to the time charts in [8] for running time comparisons of the 1 and 2-dimensional SOLF algorithms.

\section{Extensions}

In this section, we discuss extensions of the $2-D$ results. Specifically, we show that the 3 -D case of the problem can be reduced to solving a number of 2-dimensional GOPs, as opposed to 4-dimensional GOPs in [7], and thus expect significant time/space improvements in practice. The reduction to 2 -dimensional problems allows to simplify the data structures and algorithms involved in generating the feasible domains and the objective functions for various GOPs. We also mention here that, using the same models of computation as in [10], very simple parallel algorithms for generating the GOPs can be developed for the link problem, matching the time/work bounds in [10].

Similar to the 2 -D case, the 3 -D version of the weighted regions optimal link problem is a generalization of the 3 -D optimal weighted penetration problem [7]. Since a line in $3-\mathrm{D}$ can be identified using four parameters (e.g., the two spherical coordinates $(\theta, \phi)$ of the direction vector of $L$ and the projection $(u, v)$ onto the plane orthogonal to $L$ and containing the origin [11]), it is expected that in this case the optimal solution can be found by solving some 4-variable GOPs. In [7], it has been proved that finding an optimal penetration (line or semiline) can be reduced to solving $O\left(n^{4}\right)$ GOPs, by constructing the 3 -D visibility complex [11] of some transformed scene. The cells of the visibility complex can be constructed based on a direct enumeration of the vertices of the complex, followed by a sweep of these vertices. The total storage required by the visibility complex is $O\left(n^{4}\right)$ in the worst case, where $n$ is the total number of edges of the polygons in $R$, and the complex is represented using a polytope structure: each face has pointers to its boundaries (faces of lower dimension) and to the faces of larger dimension adjacent to it (see [11] for details). After constructing the visibility complex, the GOPs can be obtained by a depth-first search traversal of (the 4-D faces of) the complex [7]. For a given GOP, the feasible domain corresponds to a cell of the 
visibility complex (a $4-\mathrm{D}$ convex region) and the objective function, which is a 4-variable function defined as a sum of $k=O(n)$ fractional terms, has the form:

$$
f(u, v, \theta, \phi)=\sqrt{1+\theta^{2}+\phi^{2}} \sum_{i=1}^{k} \frac{u+a_{i} v+b_{i}}{\phi+c_{i} \theta+e_{i}}
$$

where $a_{i}, b_{i}, c_{i}$ and $e_{i}$ are constants and the variables $u, v, \theta$ and $\phi$ are the defining parameters for the link $L$. As with the $2-\mathrm{D}$ version, the case of a line segment link (case (c) of the link problem) can be reduced to that of a line, by using the data structures in $[7,9]$. We then have the following lemma.

Lemma 4. Let $f(u, v, \theta, \phi)$ be a function defined as above, over some bounded feasible domain $D$. Then, $\min _{(u, v, \theta, \phi) \in D} f(u, v, \theta, \phi)$ can be found on the $2-D$ faces (2-faces) of $D$.

As observed in [12], while valuable at a theoretical level and for some specific worst cases (e.g., grid-like scenes), the visibility complex is an intricate data structure. The algorithm that constructs the visibility complex requires to compute a 4-D subdivision and is rather complicated, and the traversal of the complex is difficult due to the multiple levels of adjacency.

From the preceding lemma, it results that it is enough to construct (and depth-first traverse) only the 2-D faces of the complex. These faces can be obtained using the visibility skeleton data structure and construction algorithm in [12]. The visibility skeleton is easy to build and its construction is based on standard geometric operations, such as line-plane intersections, which are already implemented and available as library functions (see CGAL [5], the computational geometry algorithms library, and the references therein). Once the skeleton is constructed, one can easily obtain the list of blocking objects between two specified regions (e.g., source and target) and thus the fractional terms in the corresponding objective function. Note also that, although the time and space bounds for constructing and storing the skeleton are comparable with those for the visibility complex, the experiments in [12] show its effective use for scenes with more than one thousand vertices.

\section{References}

1. L. Aleksandrov, M. Lanthier, A. Maheshwari, and J.-R. Sack, "An $\epsilon$-approximation algorithm for weighted shortest paths on polyhedral surfaces," Proc. of the 6th Scandinavian Workshop on Algorithm Theory, pp. 11-22, 1998.

2. T. Asano, L.J. Guibas and T. Tokuyama, "Walking in an arrangement topologically," Int. Journal of Computational Geometry and Applications, Vol. 4, pp. 123-151, 1994.

3. A. Brahme, "Optimization of radiation therapy," Int. Journal of Radiat. Oncol. Biol. Phys., Vol. 28, pp. 785-787, 1994.

4. L. Cai, Y. Xu and B. Zhu, "Computing the optimal bridge between two convex polygons," Information processing letters, Vol. 69, pp. 127-130, 1999. 
5. The Computational Geometry Algorithms Library, web page at http://www.cgal.org.

6. B. Chazelle and H. Edelsbrunner, "An optimal algorithm for intersecting line segments in the plane," Journal of ACM, Vol. 39, pp. 1-54, 1992.

7. D.Z. Chen, O. Daescu, X. Hu, X. Wu and J. Xu, "Determining an optimal penetration among weighted regions in two and three dimensions," Journal of Combinatorial Optimization, Spec. Issue on Optimization Problems in Medical Applications, Vol. 5, No. 1, 2001, pp. 59-79.

8. D.Z. Chen, O. Daescu, Y. Dai, N. Katoh, X. Wu and J. Xu, "Optimizing the sum of linear fractional functions and applications," Proceedings of the 11th ACM-SIAM Symposium on Discrete Algorithms, pp. 707-716, 2000.

9. O. Daescu, "On geometric optimization problems", PhD Thesis, May 2000.

10. O. Daescu, "Parallel Optimal Weighted Links," Proceedings of ICCS, Intl. Workshop on Comp. Geom. and Appl.,pp. 649-657, 2001.

11. F. Durand, G. Drettakis, and C. Puech, "The 3D visibility complex, a new approach to the problems of accurate visibility," Proc. of 7th Eurographic Workshop on Rendering, pp. 245-257, 1996.

12. F. Durand, G. Drettakis, and C. Puech, "The visibility skeleton: a powerful and efficient multi-purpose global visibility tool," Proc. ACM SIGGRAPH, pp. 89-100, 1997.

13. H. Edelsbrumner, and L.J. Guibas, "Topologically sweeping an arrangement," Journal of Computer and System Sciences, Vol. 38, pp. 165-194, 1989.

14. M.R. Garey and D.S. Johnson, "Computers and Intractability: A Guide to the Theory of NP-Completeness," W.H. Freeman, New York, NY, 1979.

15. A. Gustafsson, B.K. Lind and A. Brahme, "A generalized pencil beam algorithm for optimization of radiation therapy," Med. Phys., Vol. 21, pp. 343-356, 1994.

16. S.K. Kim and C.S. Shin, "Computing the optimal bridge between two polygons," Research Report HKUST-TCSC-1999-14, Hong-Kong University, 1999.

17. M. Lanthier, A. Maheshwari, and J.-R. Sack, "Approximating weighted shortest paths on polyhedral surfaces," Proc. of the 13th ACM Symp. on Comp. Geom., pp. 274-283, 1997.

18. C. Mata, and J.S.B. Mitchell, "A new algorithm for computing shortest paths in weighted planar subdivisions," Proc. of the 13th ACM Symp. on Comp. Geom., pp. 264-273, 1997.

19. J.S.B. Mitchell, "Geometric Shortest Paths and Network Optimization," Handbook of Computational Geometry, Elsevier Science (J.-R. Sack and J. Urrutia, eds.), 2000.

20. J.S.B. Mitchell and C.H. Papadimitriou, "The weighted region problem: Finding shortest paths through a weighted planar subdivision," Journal of ACM, Vol. 38, pp. 18-73, 1991.

21. A. Schweikard, J.R. Adler and J.C. Latombe, "Motion planning in stereotactic radiosurgery," IEEE Transactions on Robotics and Automation, Vol. 9, pp. 764-774, 1993. 